\title{
Clinical efficacy of intra-articular injections in knee osteoarthritis: a prospective randomized study comparing hyaluronic acid and betamethasone
}

This article was published in the following Dove Press journal:

Open Access Rheumatology: Research and Reviews

9 January 2015

Number of times this article has been viewed

\author{
Cesáreo Ángel Trueba \\ Davalillo ${ }^{1,2}$ \\ Cesáreo Trueba Vasavilbaso ${ }^{2}$ \\ José Mario Navarrete
}

Álvarez ${ }^{2}$

Pilar Coronel Granado 3

Ozcar Alejandro García

Jiménez ${ }^{2}$

Mercedes Gimeno del Sol ${ }^{3}$

Félix Gil Orbezo²

'School of Medicine (UNAM), México DF, Mexico; ${ }^{2}$ Orthopedic Service, Hospital Español de México, México DF, Mexico; ${ }^{3}$ Scientific Department, TEDEC-MEIJI FARMA,SA, Alcalá de Henares, Spain
Correspondence: Mercedes Gimeno del Sol

Scientific Department, TEDEC-MEIJI FARMA SA Ctra M-300, Km 30,500

Alcalá de Henares, Madrid, Spain

Emailm.gimeno@tedecmeiji.com
Background: Osteoarthritis (OA) is the most common joint disease and leading cause of disability. Intra-articular (IA) administration of hyaluronic acid (HA) or corticosteroids (CS) have been previously studied, though using insufficient number of patients or short follow-up periods.

Objective: We evaluate HA and CS in patients with knee OA in terms of clinical efficacy over 12 months.

Methods: We used a prospective, randomized study with parallel groups. Randomized patients received IA injections of HA or betamethasone (BM). The primary outcomes were improvement in pain using Visual Analog Scale and function in the Western Ontario and McMaster University Osteoarthritis Index (Likert scale). Follow-up visits were scheduled at 3 months, 6 months, 9 months, and 12 months.

Results: A total of 200 patients were included. Pain was significantly reduced in both groups at the first follow-ups. At 12 months, the mean pain reduction in the HA group was 33.6\% (95\% CI: 31.1-36.1) compared to 8.2\% (95\% CI: 5.2-11.1) in BM $(P<0.0001)$. Function improvement was higher in HA through every visit, and mean improvement at 12 months was $47.5 \%(95 \% \mathrm{CI}$ : $45.6-49.3)$ in HA patients vs $13.2 \%(95 \%$ CI: $11.4-14.9)$ in the BM group $(P<0.0001)$. All patients from both groups achieved the Minimal Clinically Important Improvement (MCII) for both pain and function up to 6 months. At 9 months and 12 months, the MCII figures were higher in HA group with $\geq 80 \%$ compared to $\leq 10 \%$ in BM group $(P<0.0001)$. Adverse reactions were rare and related to the administration procedure.

Conclusion: Both treatments effectively controlled OA symptoms. BM showed higher shortterm effectiveness, while HA showed better long-term effectiveness, maintaining clinical efficacy in a large number of patients 1 year after administration.

Keywords: viscosupplementation, corticosteroids, knee injection, joint disease

\section{Introduction}

Osteoarthritis (OA) is the most common form of joint disease and a leading cause of disability in the elderly. The main large joint affected is the knee, and a recent report ${ }^{1}$ found that symptomatic knee OA occurs in $10 \%$ of men and $13 \%$ of women aged 60 years or more.

The etiology is multifactorial, including a variety of risk factors (aging, genetics, trauma, malalignment, and obesity), which interact to cause this disorder. ${ }^{2}$ The primary objectives in OA treatment focus on pain reduction, joint mobility improvement, and functional impairment limitation. Furthermore, secondary goals are centered on the reduction of disease progression and improvement of muscular 
strength, in order to preserve patients' independence and quality of life. ${ }^{3}$

Despite the immense impact of this disease, very few effective nonsurgical options are available to handle it. Current treatment guidelines ${ }^{4-7}$ recommend starting by using nonpharmacological measures such as patient education, weight loss, and physical therapy. Nevertheless, it is commonly accepted that the optimal management of knee OA requires a combination of nonpharmacologic and pharmacologic treatments, such as acetaminophen, nonsteroidal anti-inflammatory drugs, or selective COX2 inhibitors.

An alternative to oral drugs for pain in patients with low response to analgesics and/or anti-inflammatory drugs, or with contraindications to them, is the use of intra-articular (IA) therapy. ${ }^{8}$ IA treatment is of special interest not only for pain relief and pain flares in more acute situations but also to delay any surgical intervention by improving the patients' subjective quality of life. ${ }^{9}$ Nowadays, two widely accepted products are being used for IA injections: hyaluronic acid (HA) and corticosteroids (CS).

CS knee injections have been in use for the last 60 years in the conservative management of OA, and they are recommended in several consensus documents ${ }^{4-7}$ as a useful adjunctive treatment for it. ${ }^{10} \mathrm{~A}$ major Cochrane review ${ }^{11}$ evaluated the efficacy and safety of IA CS in the treatment of knee OA. The authors concluded that it appears the beneficial effects of IA CS are fast at onset but may be relatively short-lived (1-3 weeks); in addition, there was no evidence of long-term efficacy. In spite of the numerous publications concerning the use of IA CS, there is hardly any agreement on the most efficacious dose and dosing regimen of these agents. ${ }^{12}$

Since Balazs and Delinger started their works of viscosupplementation with $\mathrm{HA},{ }^{13}$ this compound has been widely used as a nonsurgical alternative to treat OA until today. $\mathrm{HA}$ is a key molecule in joint biomechanics because of the fact that the treatment with exogenous HA contributes to the restoration of the elastic and viscous properties of the synovial fluid, resulting in pain reduction and functional improvement. Moreover, different studies have confirmed that HA interacts with inflammation mediators and matrix turnover in joint cells, reduces the apoptosis of chondrocytes, and exerts a biosynthetic-chondroprotective effect. ${ }^{14-18}$ The use of viscosupplementation is also recommended by various scientific societies. ${ }^{5-7}$

Several clinical studies have been conducted comparing face to face HA and CS in knee OA. ${ }^{19-28}$ However, very often they included a small number of patients or had a short follow-up. A meta-analysis comparing CS and HA showed a pattern of efficacy that varied over time, and concluded that, beyond 8 weeks after injection, HA had greater efficacy. ${ }^{9}$ Nevertheless, other studies, ${ }^{24,26,28}$ some of them published after the meta-analysis, did not find significant differences in clinical effects between CS and HA at 3 months or even at 6 months' follow-up, leaving open the discussion on the relative efficacy of the two products. Taking the available information into account, we found it of interest to conduct a clinical trial in order to compare HA with a CS, betamethasone (BM), evaluating both treatments in terms of clinical efficacy and enlarging the follow-up period up to 12 months.

\section{Material and methods Ethics}

The study was performed in accordance to the principles of Good Clinical Practice guidelines and in compliance with the Declaration of Helsinki. The protocol was approved by the local Ethics Review Board from Hospital Español de México, and all patients gave written informed consent to participate in the study.

\section{Design}

This was a prospective, randomized, open study with parallel groups. Patients suffering from knee OA were randomized to receive IA injections of $2.5 \mathrm{~mL}$ of $1 \% \mathrm{HA}$ with a mean molecular weight of 900,000 Da, obtained by a fermentation process from Streptococcus zoopidemicus strains (Suprahyal ${ }^{\circledR}$ ) or IA injections of BM: BM dipropionate $5.0 \mathrm{mg}+\mathrm{BM}$ sodium phosphate $2.0 \mathrm{mg}$ in $1 \mathrm{~mL}$ (Diprospan Hypack ${ }^{\circledR}$ ).

The treatment consisted of five IA injections of HA (day 0 and weekly injections afterward) or two injections of BM (day 0 and in the fourth week), and the follow-up visits were scheduled at 3 months, 6 months, 9 months, and 12 months.

Prior to inclusion, patients were assessed for fulfillment of the entry criteria, and eligible patients were informed about the study purpose and the study design. Demographic characteristics and medical history of the participants were recorded, and laboratory tests were done. X-ray of both knees was performed using anteroposterior projection with support, lateral with $30^{\circ}$ flexion and Merchant $\left(45^{\circ}\right)$ views, as well as one bipodalic mechanical axis digitalized in a single plate.

Eligible patients were randomized 1:1. In this study, a computer-generated list of random numbers was used. The random sequence was created using the freely accessible tools available at http://www.randomization.com, which uses the pseudo-random number generator of Wichmann 
and $\mathrm{Hill}^{29}$ and modified by McLeod. ${ }^{30}$ This tool allows the introduction of several treatments or intervention arms, and includes a seed for the random number generator that allows exact reproduction of the randomization schedule of the study any time and when details and labels are introduced in the same way.

The allocation sequence was concealed from the people determining the patient's eligibility at the initial visit. Once the eligibility of the patient was confirmed, a number was given, and a different person was responsible for the patient's assignment to treatment according to the randomization list. The physician in charge of evaluations at follow-up was blind to patient's group assignment, whereas the patients and the personnel administering the injections were not blinded. Administration of the IA treatments took place under aseptic conditions by inserting the needle into the patellofemoral joint space by superolateral approach, with the patients in a supine position. Arthrocentesis was performed prior to each injection in order to remove any effusion. In case of bilateral affectation, both knees were treated with the same product.

Concomitantly to the administration of IA injections, the patients of both groups initiated treatment with glucosamine sulfate $1500 \mathrm{mg}$ + meloxicam $15 \mathrm{mg}$ for 1 month. Once completed, the patients were prescribed glucosamine sulfate $1500 \mathrm{mg}$ + chondroitin sulfate $1200 \mathrm{mg}$ for one additional month. In case of continued pain during the follow-up, paracetamol was the only treatment allowed, up to $3 \mathrm{~g}$ /day.

\section{Patients selection criteria}

Eligible patients were men and women from 40 years to 85 years of age suffering from knee OA, with radiographic OA grade II-III according to Kellgren and Lawrence (KL) $)^{31}$ with a body mass index (BMI) $<35 \mathrm{~kg} / \mathrm{m}^{2}$, who had signed the informed consent form for participation.

Main exclusion criteria were a history of trauma or surgery on the target knee, inflammatory arthritis, microcrystalline arthropathies, previous unspecific knee synovitis, knee infection, angular deformity $>10^{\circ}$, and neoplasia, as well as other conditions where the administration of CS would be specifically contraindicated such as diabetes mellitus, and metabolic syndrome.

\section{Evaluation of efficacy}

The primary efficacy outcomes were reduction in global pain and function improvement using Western Ontario McMaster University Osteoarthritis (WOMAC) subscale at the end of follow-up (12 months), in comparison to baseline and the difference between both treatment groups. A 0-10 cm Visual
Analog Scale (VAS) was used for global pain measurement, and a five-point Likert scale (0-4) for WOMAC. For both VAS and Likert scales, the higher the score, the worse is the patient's condition.

The WOMAC questionnaire used was the translated version from Batlle-Gualda et al, ${ }^{32}$ validated by Escobar et al. ${ }^{33}$

Efficacy along the different visits scheduled in the protocol and consumption of acetaminophen as rescue medication for OA were assessed as secondary outcomes.

Additionally, the number of patients achieving the Minimal Clinically Important Improvement (MCII) on each treatment group was also calculated. The MCII is the smallest change in measurement that signifies an important improvement in a patient's symptom. ${ }^{34}$ In our study, we used as reference the work of Tubach et al, ${ }^{35}$ which established the MCII as the 75th percentile of the distribution of change in patient-reported outcomes scores for patients who considered they had slight or moderate improvement. The authors concluded that their work allowed promoting the use of MCII values, which were defined as 15 of 100 for absolute improvement and 20\% for relative improvement in clinical trials of rheumatic diseases, with pain, functional disability, patient global assessment, or physician global assessment used as the outcome criteria. To calculate the MCII, total scores were normalized to a $0-100$ score. $^{34}$

\section{Evaluation of safety}

The safety and tolerability of the interventions were evaluated based on the incidence and type of adverse events that could have arisen throughout the study.

\section{Statistics}

Categorical variables were summarized by their number and relative frequencies. Continuous variables following a normal distribution, mean, standard deviation, and maximum and minimum values were used to summarize. Alternatively, nonnormally distributed variables were summarized with their median, interquartile range, minimum, and maximum.

Comparisons in continuous variables between treatment groups were made using Student's $t$-test or the Mann-Whitney $\mathrm{U}$ test for normally or nonnormally distributed variables, respectively. Longitudinal changes in normally distributed variables were assessed with paired $t$-test when two time points were compared, or with ANOVA for repeated measures for more than two. If the variables were not normally distributed, the Wilcoxon signed rank sum test and Friedman's test 
were used, instead. Categorical variable comparison between treatment groups was made through the Pearson Chi-square test (Fisher's exact test when two dichotomous variables were compared).

The primary outcomes were defined as the changes from baseline in the raw scores of pain intensity and the WOMAC function subscale obtained by subtracting the follow-up visit value from the baseline value. These outcomes were analyzed with repeated measures of generalized linear models (GLM) from raw scores in order to include baseline values to avoid spurious associations.

The results of the primary outcomes were also analyzed by subgroups: age groups, sex, BMI, KL grade, and acetaminophen consumption. In all cases, a two-sided test with 95\% confidence interval was used.

The final sample size achieved a statistical power of $96.6 \%$, with a precision value of $95 \%$ (error type I equal to 0.05 ) in two groups randomized $1: 1$, to detect a difference of 1.5 points between groups compared to baseline.

The main population for analysis was the modified intention to treat (mITT) set, which included all randomized patients with at least one efficacy assessment after randomization. The last observation carried forward (LOCF) was used for handling missing data. The per protocol population (PP) was also analyzed and was composed of the patients who had completed the 12 months follow-up according to protocol. Safety analysis was performed in all patients who received at least one IA injection. Data were analyzed using the program STATA 11.2. All tests were two sided, and the statistical significance was set at 0.05 .

\section{Results}

\section{Disposition of patients}

\section{and demographic characteristics}

Patients were recruited between April 2008 and February 2011. A total of 320 patients were screened, of whom 120 $(40 \%)$ were screening failures. Five patients did not provide any efficacy data after randomization and were, therefore, not included in the analysis of efficacy, leaving a total of 195 patients in the mITT population. Finally, 89 and 91 patients receiving HA or BM, respectively, completed the study according to protocol (PP population) (Figure 1).

Within the patients, there were slightly more women (59.5\%), the mean age was 62.7 years, and mean BMI was 27.1. In reference to OA condition, there were no differences between groups regarding KL grade or the knee affected (Table 1). Significant differences were found in global pain, having higher values in the BM group $(P=0.004)$, and WOMAC in the HA group, due to higher scores in the function subscale $(P=0.001)$ (Table 2$)$.

\section{Efficacy}

In the mITT population, the raw values for pain showed a significant reduction in both groups from early follow-up (Table 2). Percentages of reduction in pain at 3 months were notably higher in the BM group (66.3\%, 95\% CI: 63.3-69.3) compared to the HA group (48.5\%, 95\% CI: 45.8-51.3) $(P<0.0001)$ (Table 3); these results showed a reversion in the following visits, with the reduction in pain being significantly higher in the HA group. At 12 months, the mean reduction in pain in the HA group was $33.6 \%$ (95\% CI: 31.1-36.1) compared to $8.2 \%(95 \% \mathrm{CI}: 5.2-11.1)$ in patients treated with BM $(P<0.0001)$. The PP population showed similar results, with the mean reduction in pain at 12 months of $34.4 \%(95 \%$ CI: 31.7-36.1) in the HA group and 7.7\% (95\% CI: 4.4-9.7) for the BM patients $(P<0.0001)$. WOMAC's total score and the subscales of pain, function, and stiffness also showed significant improvement in both treatment groups (Table 2). When the WOMAC function scores in HA and BM at different time points were analyzed, the comparison was distinctly favorable to $\mathrm{HA}$ at all visits, to the extent that, at the end of the study, patients treated with HA had a mean improvement in function of $47.5 \%$ (95\% CI: 45.6-49.3) compared to $13.2 \%$ (95\% CI: $11.4-14.9)$ in the BM group $(P<0.0001)$. In the PP population, these figures were $47.3 \%$ (95\% CI: 46.2-48.3) and $12.0 \%$ (95\% CI: 10.1-13.0), respectively $(P<0.0001)$. The comparisons between groups for WOMAC's total score, pain, and stiffness subscales followed the same pattern.

Based on the above results, the percentage of patients achieving the MCII for both pain and function was calculated. It was nearly $100 \%$ in both groups up to 6 months' follow-up. From this visit onward, the values decreased dramatically in the BM group in such a way that at 9 months the MCII for a change of at least 15 of 100 for absolute change established in the literature was $81.4 \%$ in the HA group and only $9.2 \%$ in those treated with BM $(P<0.0001)$ (Figure 2). These differences followed the same tendency when the MCII was analyzed, applying as cutoff a change of at least $20 \%$ for relative improvement: $87.6 \%$ and $10.2 \%(P<0.0001)$ for HA and $\mathrm{BM}$, respectively (Figure 3 ). The results at 12 months for a decrease of at least 15 of 100 were $77.3 \%$ and $6.1 \%$ for $\mathrm{HA}$ and $\mathrm{BM}$, respectively, and $84.5 \%$ and $5.1 \%$, respectively, when the $20 \%$ cutoff was used (Figures 2 and 3 ).

In the PP population, the MCII values when the 15 of 100 cutoff for absolute improvement was used were $82.0 \%$ for $\mathrm{HA}$ and $5.5 \%$ for BM at 9 months, and $77.5 \%$ and $2.2 \%$ 


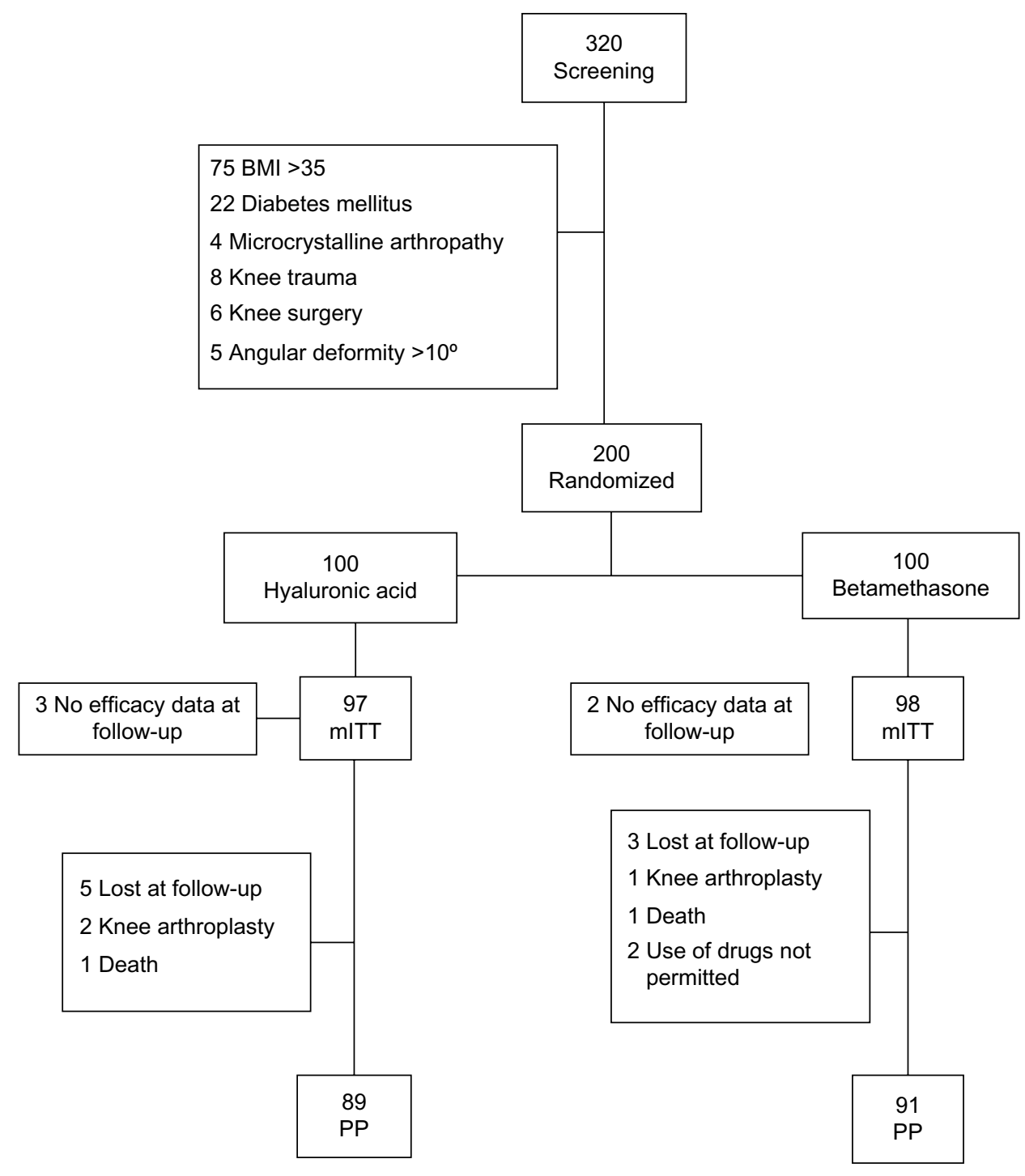

Figure I Patient disposition.

Abbreviations: BMI, body mass index; mITT, modified intention to treat; PP, protocol population.

at 12 months for HA and BM, respectively $(P<0.0001)$. When the cutoff was $20 \%$ for relative improvement, the values were $88.8 \%$ for $\mathrm{HA}$ and $6.6 \%$ for BM at 9 months and $85.4 \%$ and $1.1 \%$ at 12 months, for HA and BM, respectively $(P<0.0001)$.

The main efficacy variables (change vs baseline in global pain and WOMAC function) were analyzed using a GLM to control the effect of baseline variables that resulted in being significant, such as BMI. The resulting model for changes in global pain was adjusted by time, treatment group, BMI, and age (the last one was only maintained for the absolute difference). In the mITT population, the odds ratio (OR) for MCII varied from 105.508 (95\% CI: $22.572-493.176)$ to $73.449(95 \%$ CI: 16.345-330.052) for absolute improvement (defined as 15 of 100 change) and from 329.603 (95\% CI: 49.848-2179.375) to 243.594 (95\% CI: $37.258-1592.648$ ) for relative improvement (defined as $20 \%$ change). In the PP population, these figures varied from 98.514 (95\% CI: 22.327-434.676) to 64.321 (95\% CI: 16.14-256.33) for absolute improvement and from 249.445 (95\% CI: 43.107-1443.438) to 160.3 (95\% CI: 30.8-834.5) for relative improvement. BMI was not significant for models considering the WOMAC function subscale.

Overall, $67.4 \%$ of patients in the mITT population and $70.6 \%$ in PP took acetaminophen as rescue medication during the follow-up period, with no differences between groups.

\section{Safety}

Adverse reactions were all related to the administration procedure, and experienced by $3.5 \%$ of the patients: six cases 
Table I Demographic and osteoarthritis baseline characteristics of study groups

\begin{tabular}{|c|c|c|c|c|c|}
\hline & \multicolumn{2}{|l|}{ HA $(n=97)$} & \multicolumn{2}{|l|}{ BM $(n=98)$} & \multirow[t]{2}{*}{$P$-value } \\
\hline & Mean (SD) & n (\%) & Mean (SD) & n (\%) & \\
\hline \multicolumn{6}{|l|}{ Demographic characteristics } \\
\hline Women & & $59(60.8)$ & & $57(58.2)$ & ns \\
\hline Age, years & $62.7(0.6)$ & & $62.8(0.6)$ & & ns \\
\hline Age, distribution & & & & & ns \\
\hline$<65$ years & & $66(68.0)$ & & $64(65.3)$ & \\
\hline$>65$ years & & $31(32.0)$ & & $34(34.7)$ & \\
\hline BMI $\left(\mathrm{kg} / \mathrm{m}^{2}\right)$ & $28.3(0.5)$ & & $26.3(0.4)$ & & 0.002 \\
\hline BMI, distribution & & & & & 0.038 \\
\hline Normal weight & & $23(23.7)$ & & $36(36.7)$ & \\
\hline Overweight & & $35(36.1)$ & & $38(38.8)$ & \\
\hline Obesity type I & & $39(40.2)$ & & $24(24.5)$ & \\
\hline \multicolumn{6}{|l|}{ OA baseline characteristics } \\
\hline Kellgren-Lawrence Grade, n (\%) & & & & & ns \\
\hline II & & $62(63.9)$ & & $65(66.6)$ & \\
\hline III & & $35(36.1)$ & & $33(33.7)$ & \\
\hline Painful knee n (\%) & & & & & ns \\
\hline Right & & $45(46.4)$ & & $48(49.0)$ & \\
\hline Left & & $40(41.2)$ & & $38(38.8)$ & \\
\hline Both & & $12(12.4)$ & & $12(12.2)$ & \\
\hline
\end{tabular}

Abbreviations: BM, betamethasone; BMI, body mass index; HA, hyaluronic acid; ns, nonsignificant; OA, osteoarthritis; SD, standard deviation.

of pain (four in the group treated with HA and two in BM) and one erythema in the HA group. Effusion was detected in $3.5 \%$ of the patients (five patients in the HA group) when attending the second (three patients), third (one patient), and fifth (one patient) injection, and two in the BM group when attending for the second injection.

\section{Discussion}

This clinical trial comparing HA and BM showed remarkable long-term improvement in knee OA symptoms after treatment in both groups, with statistical and clinical differences favoring HA $(P<0.0001)$.

Since Hollander reported great clinical response to IA hydrocortisone injections in the $1950 \mathrm{~s},{ }^{36,37}$ IA CS injections have become a notable rheumatologic practice for the management of articular and periarticular inflammatory and pain conditions. Several formulations of CS are used in clinical practice, and though, on one hand, numerous investigations have been conducted, on the other, there is a lack of consensus in identifying variables such as the optimal CS agent or the dose and dosing frequency for OA treatment. ${ }^{11,38}$ The preferences vary geographically without a clear rationale, ${ }^{38}$ and current usage patterns are very often determined by the individual practitioner's opinion. ${ }^{11}$

The IA HA injections for knee OA have only been used in humans during the last 30 years, and have become widely employed since the early 1990s. ${ }^{39}$ Even though during this time multiple HA efficacy studies have been performed, studies with more than 6 months follow-up periods are rare, and, to our knowledge, there is only one clinical trial showing the carry-over effect of HA after repeated cycles of injections, lasting for at least 1 year. ${ }^{40}$

Several studies in knee OA have been carried out in order to compare the effects of IA HA and CS. These studies have found favorable results with both treatments, but very often they had short follow-up periods, making it difficult to rate one method above the other. A recent meta-analysis concluded that, from baseline to week 4, CS appeared to be relatively more effective in pain relief than $\mathrm{HA}$, but beyond week 8 , HA showed greater efficacy. ${ }^{9}$ Nevertheless, we found out that some studies, with up to 6 months follow-up and conducted with HAs of different molecular weight, did not find differences between both treatments at the end of the study. ${ }^{24,26,28}$ Consequently, our work had a double purpose: the evaluation of both treatments over a long follow-up period, and analysis of the results in terms of not only statistical differences but also clinical significance. In our study and from the first evaluation visit after the interventions, we found a significant improvement in both groups compared to baseline in terms of VAS pain and WOMAC score. At 3 months, the data suggest that BM group had greater reduction in global pain while all WOMAC subscales including pain suggest greater improvement in the HA group compared to the BM group. As for the higher reduction in global pain observed in the BM group, it has been reported that CS have a short-term effect on pain but have no effect on function, 


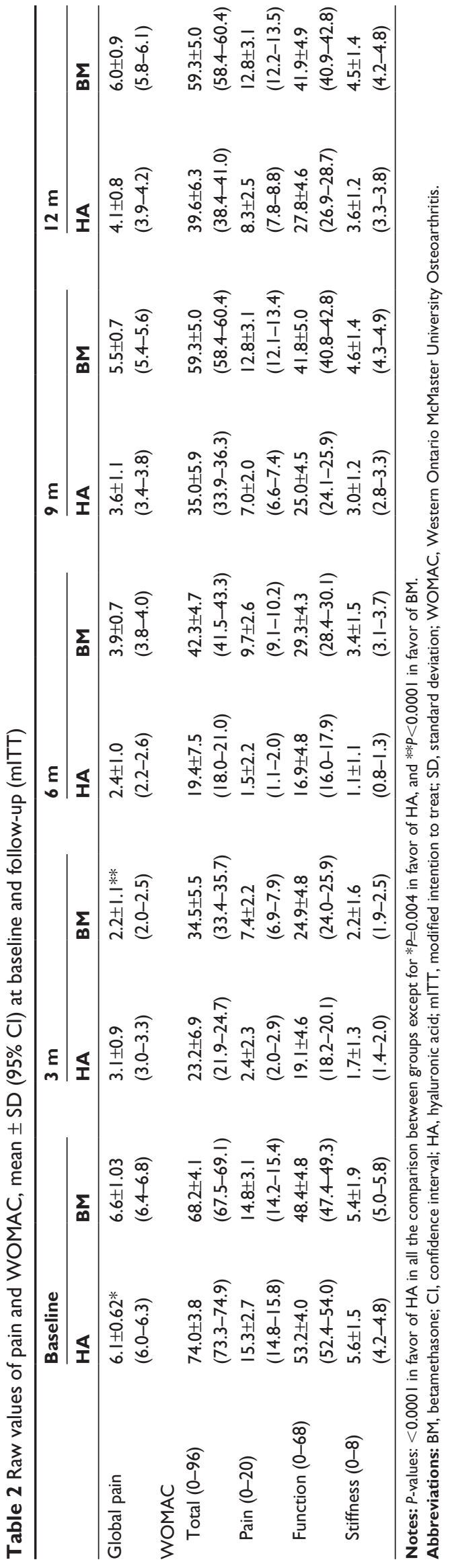

whereas HA products can provide a more durable response with relief of pain and improvement in function, although the onset of these effects is slower. ${ }^{10}$ In this sense, our results would be in line with the behavior that could be expected for both products. On the other hand, it has been reported that the WOMAC pain subscale is highly related to physical function, ${ }^{41,42}$ so it does not seem strange that WOMAC pain goes on the same line as WOMAC function subscale, and differences with global pain should not be disregarded, as occurred in our study.

From this point (3 months) onward, the efficacy of BM decreased and resulted in significant statistical differences in favor of HA, which continued up to the end of the study.

When the results were also analyzed in terms of MCII by applying the cutoffs for absolute and relative change for pain and function, we did not find any difference between groups up to the 6 months follow-up, which could be in line with the studies mentioned that found longer efficacy periods for CS. Nevertheless, from this visit onward, the values in the BM group showed a dramatic decrease to the extent that at 9 months the percentage of patients showing an MCII for pain and function were $87.6 \%$ in the HA group and only $10.2 \%$ for BM $(P<0.0001)$. The results in the HA group remained constant at 12 months.

Previous studies and meta-analyses have shown the efficacy versus placebo of $\mathrm{HA}^{43-45}$ or $\mathrm{CS}^{10}$ or have compared both treatments in the short term. ${ }^{19-28}$ The aim of this study was to compare the efficacy of both treatments in the long term and using a different approach. To the best of our knowledge, this is the first study to compare CS and HA in terms of clinical improvement. The importance of defining a minimum important improvement to use in clinical trials is widely accepted. This approach will be most helpful when interpreting the results and addition of useful information for daily practice in order to provide information that is complementary to the conventional presentation of results at the group level (mean changes in scores). ${ }^{35}$

Like any research work, our work has some limitations and it is important to mention them. First, BMI was significantly higher in the HA group, with more obesity type I patients, so a priori this could prejudice the results obtained in this group. The GLM results showed that function was not affected, whereas with reference to pain the GLM analysis showed a positive effect of HA, which, a priori, could be considered a contradiction. Secondly, the participating patients showed differences in some OA symptoms at baseline. In our opinion, in both cases the large differences found between groups in all the comparisons 
Table 3 Mean percentage of improvement expressed as percentage of decrease in the score relative to baseline \pm SD (95\% Cl) in Global Pain and WOMAC (mITT)

\begin{tabular}{|c|c|c|c|c|c|c|c|c|}
\hline & \multicolumn{2}{|l|}{$3 \mathrm{~m}$} & \multicolumn{2}{|l|}{$6 \mathrm{~m}$} & \multicolumn{2}{|l|}{$9 \mathrm{~m}$} & \multicolumn{2}{|l|}{$12 \mathrm{~m}$} \\
\hline & HA & BM & HA & BM & HA & BM & HA & BM \\
\hline Global pain & $\begin{array}{l}48.5 \pm 13.5 \\
(45.8-51.3)\end{array}$ & $\begin{array}{l}66.3 \pm 15.0^{*} \\
(63.3-69.3)\end{array}$ & $\begin{array}{l}60.6 \pm 14.8 \\
(57.6-63.6)\end{array}$ & $\begin{array}{l}39.6 \pm 12.6 \\
(37.0-42.1)\end{array}$ & $\begin{array}{l}41.2 \pm 16.4 \\
(37.9-44.5)\end{array}$ & $\begin{array}{l}14.4 \pm 17.8 \\
(10.8-17.9)\end{array}$ & $\begin{array}{l}33.6 \pm 12.4 \\
(31.1-36.1)\end{array}$ & $\begin{array}{l}8.2 \pm 4.8 \\
(5.2-11.1)\end{array}$ \\
\hline \multicolumn{9}{|l|}{ WOMAC } \\
\hline Total & $\begin{array}{l}68.6 \pm 8.9 \\
(66.8-70.4)\end{array}$ & $\begin{array}{l}49.5 \pm 5.4 \\
(48.4-50.6)\end{array}$ & $\begin{array}{l}73.7 \pm 9.7 \\
(71.7-75.7)\end{array}$ & $\begin{array}{l}37.9 \pm 5.2 \\
(36.8-38.9)\end{array}$ & $\begin{array}{l}52.6 \pm 7.9 \\
(51.0-54.2)\end{array}$ & $\begin{array}{l}12.9 \pm 6.9 \\
(|1.4-| 4.2)\end{array}$ & $\begin{array}{l}46.2 \pm 9.3 \\
(44.3-48.1)\end{array}$ & $\begin{array}{l}12.9 \pm 6.2 \\
(11.6-\mid 4.2)\end{array}$ \\
\hline Pain & $\begin{array}{l}83.7 \pm 15.7 \\
(80.5-86.8)\end{array}$ & $\begin{array}{l}49.9 \pm 14.8 \\
(47.0-52.9)\end{array}$ & $\begin{array}{l}90.0 \pm 14.8 \\
(87.0-93.0)\end{array}$ & $\begin{array}{l}33.3 \pm 17.9 \\
(29.7-36.9)\end{array}$ & $\begin{array}{l}52.8 \pm I 5.7 \\
(49.6-55.9)\end{array}$ & $\begin{array}{l}|3.2 \pm| 4.0 \\
(10.4-16.0)\end{array}$ & $\begin{array}{l}44.1 \pm 19.8 \\
(40.1-48.1)\end{array}$ & $\begin{array}{l}|2.7 \pm| 4.3 \\
(9.8-\mid 5.6)\end{array}$ \\
\hline Function & $\begin{array}{l}63.9 \pm 8.7 \\
(62.1-65.6)\end{array}$ & $\begin{array}{l}48.4 \pm 7.9 \\
(46.8-50.0)\end{array}$ & $\begin{array}{l}68.1 \pm 8.8 \\
(66.3-69.8)\end{array}$ & $\begin{array}{l}39.2 \pm 7.8 \\
(37.7-40.8)\end{array}$ & $\begin{array}{l}52.8 \pm 8.6 \\
(51.1-54.6)\end{array}$ & $\begin{array}{l}13.2 \pm 9.9 \\
(11.2-15.1)\end{array}$ & $\begin{array}{l}47.5 \pm 9.2 \\
(45.6-49.3)\end{array}$ & $\begin{array}{l}13.2 \pm 8.8 \\
(\mid 1.4-14.9)\end{array}$ \\
\hline Stiffness & $\begin{array}{l}67.6 \pm 27.5 \\
(62.1-73.2)\end{array}$ & $\begin{array}{l}57.5 \pm 28.9 * * \\
(51.7-63.3)\end{array}$ & $\begin{array}{l}79.5 \pm 23.6 \\
(74.7-84.2)\end{array}$ & $\begin{array}{l}28.7 \pm 49.9 \\
(18.7-38.7)\end{array}$ & $\begin{array}{l}44.3 \pm 22.3 \\
(39.8-48.8)\end{array}$ & $\begin{array}{l}6.5 \pm 4.8 \\
(-2.5-15.5)\end{array}$ & $\begin{array}{l}33.8 \pm 22.3 \\
(29.3-38.3)\end{array}$ & $\begin{array}{l}10.5 \pm 33.8 \\
(3.7-17.3)\end{array}$ \\
\hline
\end{tabular}

Notes: $P$-values: $<0.0001$ in favor of HA in all the comparison between groups except for $* P<0.0001$ in favor of $B M$, $* * P<0.012$ in favor of $\mathrm{HA}$.

Abbreviations: BM, betamethasone; $\mathrm{Cl}$, confidence interval; HA, hyaluronic acid; mITT, modified intention to treat; SD, standard deviation; WOMAC, Western Ontario McMaster University Osteoarthritis.

made during follow-ups and the consistency of the results across the study minimize the possible risk of bias in their interpretation.

Overall, a significant number of patients (67.4\%) took acetaminophen as rescue medication during the follow-up period, with no differences between groups. The patients were asked at every assessment visit about the consumption, and contingency analyses within group and between groups concluded that the use of rescue medication did not interfere with the assessment of the clinical outcome. The administration of glucosamine and meloxicam for 1 month, and thereafter glucosamine and chondroitin for one additional month concomitantly to IA injections, could be a limitation to our study. This procedure is based on our usual clinical practice, and it was decided to keep this scheme in the protocol as well, in addition to the study interventions. However, these

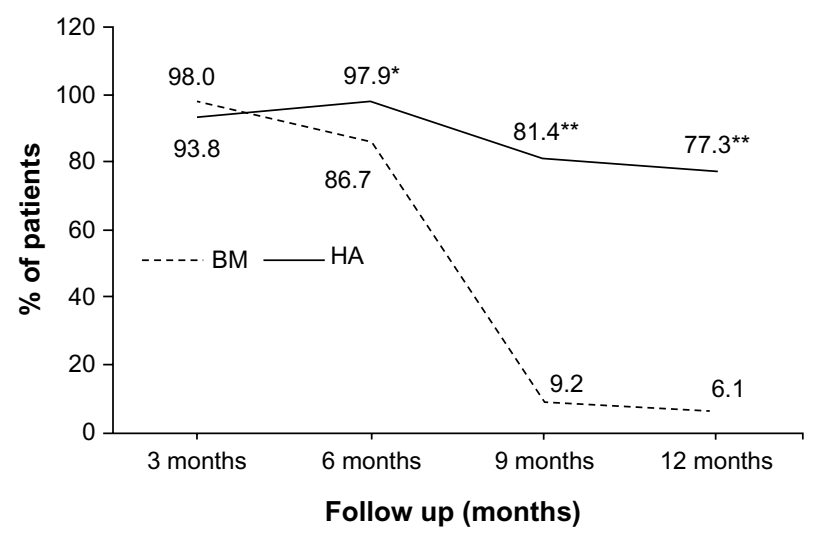

Figure 2 Percentage of patients with MCll ( 15 points) in pain and function during the study by treatment group ( $\mathrm{mlTT}$ ) ( $\mathrm{n}=97$ patients in HA and $n=98$ in BM at all time points).

Note: $* P=0.003 ; * * P<0.001$.

Abbreviations: BM, betamethasone; HA, hyaluronic acid; m, months; MClI, Minimal Clinically Important Improvement; mITT, modified intention to treat. treatments were administered to all patients, and the time elapsed between the discontinuation of the treatments and the first evaluation visit was considered sufficient.

Adverse reactions were rare and related to the administration procedure, concluding that both treatments were safe and well tolerated, in accordance with other publications. ${ }^{10,38,43,44}$ A recent meta-analysis ${ }^{46}$ showed negative conclusions about the safety profile of HA in knee OA. Such conclusions result from a questionable selection of the evidence; the methodological procedures used in the analyses of safety have been also questioned in a later review. ${ }^{47}$ This review found that only two serious adverse events from only one ${ }^{48}$ of the studies analyzed (out of 71 studies) should be reported as related to HA. Moreover, the task force of the European Society for Clinical and Economic Aspects of Osteoporosis and Osteoarthritis (ESCEO) ${ }^{7}$ does not endorse the negative results of the meta-analysis. With the current data, it can be

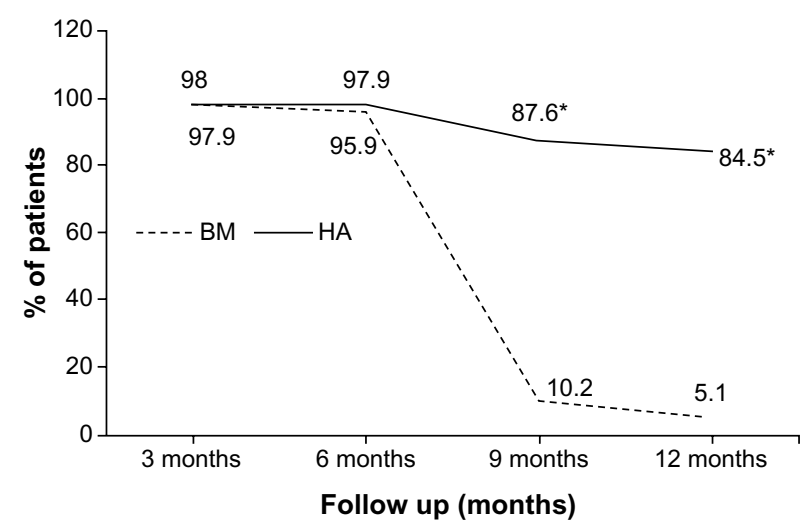

Figure 3 Percentage of patients with MCII (20\%) in pain and function during the study by treatment group ( $\mathrm{mlTT}$ ) ( $\mathrm{n}=97$ patients in $\mathrm{HA}$ and $\mathrm{n}=98$ in BM at all time points). Note: $* P<0.001$.

Abbreviations: BM, betamethasone; HA, hyaluronic acid; m, months; MCII, Minimal Clinically Important Improvement; mITT, modified intention to treat. 
concluded that intra-articular injections of HA are safe and do not place the individuals at risk.

The results of our study show that both treatments are effective in controlling OA symptoms but the pattern varies over time. The two treatments showed equal efficacy at initial follow-ups, but HA demonstrated a clearly superior long-term effectiveness than BM, with sustained clinical efficacy levels in a significant number of patients 1 year after administration.

\section{Acknowledgments}

The authors would like to thank to Ignacio Méndez, Noelia Alfaro, and Belén San José from OXON Epidemiology Ltd for the statistical analysis of the results.

\section{Disclosure}

The authors report no conflicts of interest in this work.

\section{References}

1. Zhang Y, Jordan JM. Epidemiology of osteoarthritis. Clin Geriatr Med. 2010;26:355-369.

2. Loeser RF. Age-related changes in the musculoskeletal system and the development of osteoarthritis. Clin Geriatr Med. 2010;26:371-386.

3. Snibbe JC, Gambardella RA. Treatment options for osteoarthritis. Orthopedics. 2005;28(2 Suppl):S215-S220.

4. Brown GA. AAOS clinical practice guideline: treatment of osteoarthritis of the knee: evidence-based guideline. J Am Acad Orthop Surg. 2013;21(9):577-579.

5. Hochberg MC, Altman RD, April KT, et al; American College of Rheumatology. American College of Rheumatology 2012 recommendations for the use of nonpharmacologic and pharmacologic therapies in osteoarthritis of the hand, hip, and knee. Arthritis Care Res (Hoboken). 2012;64(4):465-474.

6. Zhang W, Nuki G, Moskowitz RW, et al. OARSI recommendations for the management of hip and knee osteoarthritis: part III: changes in evidence following systematic cumulative update of research published through January 2009. Osteoarthritis Cartilage. 2010;18(4):476-499.

7. Bruyère $\mathrm{O}$, Cooper $\mathrm{C}$, Pelletier $\mathrm{JP}$, et al. An algorithm recommendation for the management of knee osteoarthritis in Europe and internationally: a report from a task force of the European Society for Clinical and Economic Aspects of Osteoporosis and Osteoarthritis (ESCEO). Semin Arthritis Rheum. 2014;44(3):253-263.

8. Neustadt DH. Intra-articular injections for osteoarthritis of the knee. Cleve Clin J Med. 2006;73(10):897-898.

9. Colen S, van den Bekerom MP, Bellemans J, Mulier M. Comparison of intra-articular injections of hyaluronic acid and corticosteroid in the treatment of osteoarthritis of the hip in comparison with intra-articular injections of bupivacaine. Design of a prospective, randomized, controlled study with blinding of the patients and outcome assessors. $B M C$ Musculoskelet Disord. 2010;11:264.

10. Bannuru RR, Natov NS, Obadan IE, Price LL, Schmid CH, McAlindon TE. Therapeutic trajectory of hyaluronic acid versus corticosteroids in the treatment of knee osteoarthritis: a systematic review and meta-analysis. Arthritis Rheum. 2009;61(12):1704-1711.

11. Bellamy N, Campbell J, Robinson V, Gee T, Bourne R, Wells G. Intraarticular corticosteroid for treatment of osteoarthritis of the knee. Cochrane Database Syst Rev. 2006;19:CD005328.

12. Douglas RJ. Corticosteroid injection into the osteoarthritic knee: drug selection, dose, and injection frequency. Int J Clin Pract. 2012;66(7):699-704.

13. Balazs EA, Delinger JL. Viscosupplementation: a new concept in the treatment of osteoarthritis. J Rheumatol Suppl. 1993;39:3-9.
14. Yu CJ, Ko CJ, Hsieh CH, et al. Proteomic analysis of osteoarthritic chondrocyte reveals the hyaluronic acid-regulated proteins involved in chondroprotective effect under oxidative stress. J Proteomics. 2014;99:40-53.

15. Peng H, Zhou JL, Liu SQ, Hu QJ, Ming JH, Qiu B. Hyaluronic acid inhibits nitric oxide-induced apoptosis and dedifferentiation of articular chondrocytes in vitro. Inflamm Res. 2010;59(7):519-530.

16. Waddell DD, Kolomytkin OV, Dunn S, Marino AA. Hyaluronan suppresses IL-1beta-induced metalloproteinase activity from synovial tissue. Clin Orthop Relat Res. 2007;465:241-248.

17. Greenberg DD, Stoker A, Kane S, Cockrell M, Cook JL. Biochemical effects of two different hyaluronic acid products in a co-culture model of osteoarthritis. Osteoarthritis Cartilage. 2006;14(8):814-822.

18. Karna E, Miltyk W, Pałka JA, Jarzabek K, Wołczyński S. Hyaluronic acid counteracts interleukin-1-induced inhibition of collagen biosynthesis in cultured human chondrocytes. Pharmacol Res. 2006;54(4): $275-281$.

19. Leardini G, Mattara M, Franceschini M, Perbellini A. Intra-articular treatment of knee osteoarthritis. A comparative study between hyaluronic acid and 6-methyl prednisolone acetate. Clin Exp Rheumatol. 1991;9:375-381.

20. Pietrogrande V, Melanotte PL, D'Agnolo B, Ulivi M, Benigni GA, Turchetto L. Hyaluronic acid versus methyl prednisolone intra-articularly injected for treatment of osteoarthritis of the knee. Curr Ther Res. 1991;50(5):670-691.

21. Jones AC, Pattrick M, Doherty S, Doherty M. Intra-articular hyaluronic acid compared to intra-articular triamcinolone hexacetonide in inflammatory knee osteoarthritis. Osteoarthritis Cartilage. 1995;3: 269-273.

22. Frizziero L, Pasquali Ronchetti I. Intra-articular treatment of osteoarthritis of the knee: an arthroscopic and clinical comparison between hyaluronic acid (500-730 kDa) and methylprednisolone acetate. J Orthop Traumatol. 2002;3:89-96.

23. Tascioglu F, Oner C. Efficacy of intra-articular sodium hyaluroante in the treatment of knee osteoarthritis. Clin Rheumatol. 2003;22: 112-117.

24. Leopold SS, Redd BB, Warme WJ, Wehrle PA, Pettis PD, Shott S Corticosteroid compared with hyaluronic acid injections for the treatment of osteoarthritis of the knee. A prospective, randomized trial. J Bone Joint Surg Am. 2003;85-A(7):1197-1203.

25. Caborn D, Rush J, Lanzer W, Parenti D, Murray C. A randomized, single-blind comparison of the efficacy and tolerability of hylan G-F 20 and triamcinolone hexacetonide in patients with osteoarthritis of the knee. J Rheumatol. 2004;31:333-343.

26. Shimizu M, Higuchi H, Takagishi K, Shinozaki T, Kobayashi T. Clinical and biochemical characteristics after intra-articular injection for the treatment of osteoarthritis of the knee: prospective randomized study of sodium hyaluronate and corticosteroid. J Orthop Sci. 2010;15(1):51-56

27. Skwara A, Ponelis R, Tibesku CO, Rosenbaum D, Fuchs-Winkelmann S. Gait patterns after intraarticular treatment of patients with osteoarthritis of the knee - hyaluronan versus triamcinolone: a prospective, randomized, double blind, monocentric study. Eur J Med Res. 2009;14(4): $157-164$.

28. Leighton R, Akermark C, Therrien R, et al; DUROLANE Study Group. NASHA hyaluronic acid vs methylprednisolone for knee osteoarthritis: a prospective, multi-centre, randomized, non-inferiority trial. Osteoarthritis Cartilage. 2014;22(1):17-25.

29. Wichmann BA, Hill ID. Algorithm AS 183. An efficient and portable pseudo-random number generator. Appl Stat. 1982;31(2):188-190.

30. McLeod AI. Remark AS R58: a remark on algorithm AS 183. An efficient and portable pseudo-random number generator. Appl Stat. 1985;34(2):198-200.

31. Kellgren JH, Lawrence JS. Radiological assessment of osteoarthritis. Ann Rheum Dis. 1957;16:494-501.

32. Batlle-Gualda E, Esteve-Vives J, Piera Riera MC, Hargreaves R, Cutts J. Traducción y adaptación al español del cuestionario WOMAC específico para artrosis de rodilla y cadera [Translation and adaptation to Spanish of the WOMAC questionnaire specific for knee and hip osteoarthritis]. Rev Esp Reumatol. 1999;26:38-45. Spanish. 
33. Escobar A, Quintana JM, Bilbao A, Azkárate J, Güeaga JI. Validation of the Spanish version of the WOMAC questionnaire for patients with hip or knee osteoarthritis. Western Ontario and McMaster Universities Osteoarthritis Index. Clin Rheumatol. 2002;21(6):466-471.

34. Tubach F, Ravaud P, Baron G, et al. Evaluation of clinically relevant changes in patient reported outcomes in knee and hip osteoarthritis: the minimal clinically important improvement. Ann Rheum Dis. 2005;64(1): 29-33.

35. Tubach F, Ravaud P, Martin-Mola E, et al. Minimum clinically important improvement and patient acceptable symptom state in pain and function in rheumatoid arthritis, ankylosing spondylitis, chronic back pain, hand osteoarthritis, and hip and knee osteoarthritis: results from a prospective multinational study. Arthritis Care Res (Hoboken). 2012;64(11):1699-1707.

36. Hollander JL. Intra-articular hydrocortisone in arthritis and allied conditions; a summary of two years' clinical experience. J Bone Joint Surg Am. 1953;35-A:983-990.

37. Hollander JL, Jessar RA, Brown EM Jr. Intra-synovial corticosteroid therapy: a decade of use. Bull Rheum Dis. 1961;11:239-240.

38. Garg N, Perry L, Deodhar A. Intra-articular and soft tissue injections, a systematic review of relative efficacy of various corticosteroids. Clin Rheumatol. 2014;33(12):1695-1706.

39. Bert JM, Bert TM. Nonoperative treatment of unicompartmental arthritis. From bracing to injection. Clin Sports Med. 2014;33:1-10.

40. Navarro-Sarabia F, Coronel P, Collantes E, et al; AMELIA study group. A 40-month multicentre, randomised placebo-controlled study to asses the efficacy and carry-over effect of repeated intra-articular injections of hyaluronic acid in knee osteoarthritis: the AMELIA project. Ann Rheum Dis. 2011;70(11):1957-1962.
41. Thumboo J, Chew LH, Soh CH. Validation of the Western Ontario and McMaster University Osteoarthritis Index in Asians with osteoarthritis in Singapore. Osteoarthritis Cartilage. 2001;9(5):440-446.

42. Gandek B. Measurement properties of the Western Ontario and McMaster Universities Osteoarthritis Index: a systematic review. Arthritis Care Res. Epub 2014 July 21.

43. Miller LE. US-approved intra-articular hyaluronic acid injections are safe and effective in patients with knee osteoarthritis: systematic review and meta-analysis of randomized, saline-controlled trials. Clin Med Insigths Arthritis Musculoskelet Disord. 2013;6:57-63.

44. Bellamy N, Campbell J, Robinson V, Gee T, Bourne R, Wells G. Viscosupplementation for the treatment of osteoarthritis of the knee. Cochrane Database Syst Rev. 2006;2:CD005321.

45. Bannuru RR, Natov NS, Dasi UR, Schmid CH, McAlindon TE. Therapeutic trajectory following intra-articular hyaluronic acid injection in knee osteoarthritis - meta-analysis. Osteoarthritis Cartilage. 2011;19(6):611-619.

46. Rutjes AW, Jüni $P$, da Costa BR, Trelle $S$, Nüesch E, Reichenbach S. Viscosupplementation for osteoarthritis of the knee: a systematic review and meta-analysis. Ann Intern Med. 2012;157(3):180-191.

47. McAlindon TE, Bannuru RR. Osteoarthritis: is viscosupplementation really so unsafe for knee OA? Nat Rev Rheumatol. 2012;8(11): 635-636.

48. Huskisson EC, Donnelly S. Hyaluronic acid in the treatment of osteoarthritis of the knee. Rheumatology (Oxford). 1999;38(7):602-607.

\section{Publish your work in this journal}

Open Access Rheumatology Research and Reviews is an international, peer-reviewed, open access journal, publishing all aspects of clinical and experimental rheumatology in the clinic and laboratory including the following topics: Pathology, pathophysiology of rheumatological diseases; Investigation, treatment and management of rheumatological

\section{Dovepress}

diseases; Clinical trials and novel pharmacological approaches for the treatment of rheumatological disorders. The manuscript management system is completely online and includes a very quick and fair peerreview system, which is all easy to use. Visit http://www.dovepress.com/ testimonials.php to read real quotes from published authors. 